\title{
Motivation-Based Game Design: A Framework for Evaluating Engagement Potential
}

\author{
Charles Butler \\ The Norwegian School of Information Technology, Oslo, Norway \\ charlesabutler@gmail.com
}

\begin{abstract}
Video games excel at capturing and maintaining the interest of players all around the world. However, actually moving a game from an initial concept to an attention-grabbing best-seller is quite a difficult and unlikely event. Even though the best games seem to have an almost magical ability to generate engagement among players, the vast majority of games fall far short of this mark. Likewise, many serious games and gamified products have considerable difficulty achieving their desired levels of engagement. Success with in these areas can be even more elusive as they are burdened with some disadvantages of traditional games without being able to leverage all of the advantages. This paper presents a motivation-based framework intended to evaluate the engagement potential of a game design, whether it be a traditional game, serious game, or gamified product.
\end{abstract}

Keywords: game design, game mechanics, gamification, motivation, serious games.

\section{Introduction}

Video games are often labeled (both positively and negatively, depending on the audience) with terms describing the duration and/or intensity of the engagement that they create, terms such as immersive, stimulating, or addictive. These traits are typi-cally very desirable from the perspective of the game's creators, and they can, at least in part, determine the extent to which a game is successful. Judging by the low percentage of commercial video games which actually turn out to be profitable, we can confidently say that achieving a sufficient level of engagement is a tremendously difficult endeavor, even for professional game studios with ever-increasing budgets which are at time approaching or even exceeding $\$ 100$ million.

If massive commercial studios funded or owned by the largest video game publishing companies in the world have trouble with engagement, then consider how the makers of educational games, serious games, or gamified products could hope to compete when they are likely to have access to far fewer resources. To compound the difficulty, these alternative games tend to suffer similar difficulties to traditional vid-eo games (complexity, difficulty estimating proper schedules and budgets, etc.) as well as the additional difficulties that arise when making a 
non-entertainment-based product (less appealing subject material, low production quality when compared to the expectations that are set by traditional video games, creators are often subject matter experts as opposed to game design experts, etc.).

Despite these difficulties, this paper presents a framework for evaluating a game's engagement potential by mapping player motivations to the various game mechanics being utilized by the game. In addition, this framework can be used to pinpoint areas where a game could add or modify mechanics in order to increase its engagement potential. Of course, this doesn't directly address all of the issues (ever-increasing production values and their corresponding budgets, for example), but it can help a team with limited resources focus their efforts in order to achieve the best results from the work that its members are able to do.

\section{Motivation}

\subsection{Motivation Frameworks}

There have been prior attempts at creating a model of player motivations. Bartle's work, for example, attempts to identify the motivations that guide a player's action within a game context. Starting with a 4 point model and later evolving into an 8 point model, Bartle asserts that different types of players want different things from their games and that by intentionally planning a game's features around a set of assumed player types, the developer could control, to some extent, the mix of player types actively using the game.

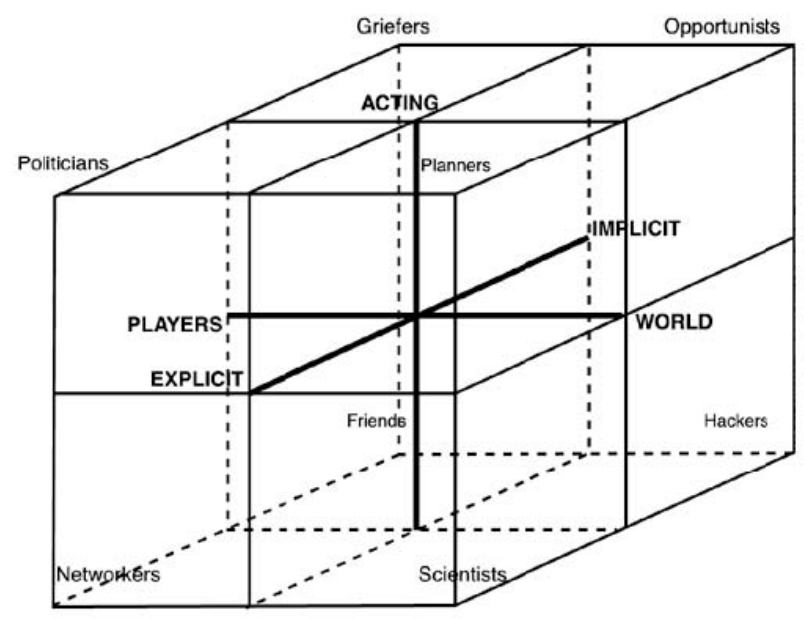

Fig. 1. Player Types (Bartle 2003) 


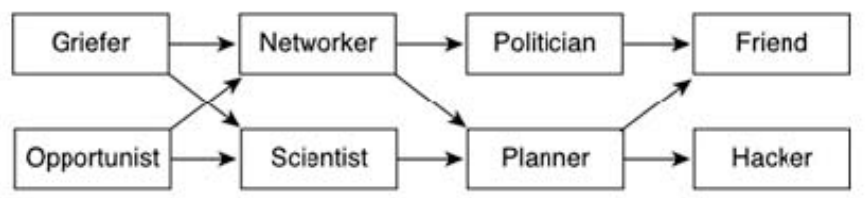

Fig. 2. Player Type Progression (Bartle 2003)

However, these models deal primarily behind in-game motivations and their resulting behaviors. This keeps the model at least one step removed from the basic human emotions that drive all of our day-to-day behaviors, whether we're playing a game at home, performing a task at work, or basically any behavior in any setting. To remedy this, I instead want to look at game features and/or mechanics through a lens which addresses the fundamentals of human behavior. The perspective chosed for this model is Maslow's Heirarchy of Needs, which addresses and prioritizes our innate human needs.

\subsection{Driving Engagement}

A recurring issue that I've noticed with less successful commercial games as well as with most educational/serious games (and to an even greater extent with gamifica-tion attempts) is that they tend to be more focused on the creator's objectives than on the players'. If a game isn't created with the users motivations in mind, it shouldn't be surprising when it fails to sufficiently engage them. The simple theory is that the more types of needs and motivations that a game can incorporate, the higher the chance of the game being especially engaging to a given player. The purpose of creating a framework is to allow developers to if their game is addressing a sufficient range of motivations and to help identify areas where it could more effectively do so.

However, the challenge is that even when using the framework, the path of least resistance will lead many well-intentioned developers into excessively superficial feature designs in order to cover as much of the motivational roadmap as possible. Unless a feature has a certain amount of depth, the engagement that it creates with either be short-lived or of negligible intensity.

\subsection{Evaluation}

When looking at any game design, we should evaluate it based on both the number of ways in which the game could help fulfill a particular need or motivation. In addi-tion, we should also consider to what extent that need could be filled.

In considering Maslow's Hierarchy of Needs, we can likely disregard the base level of physiological needs, but games are capable of providing at least some elements in the remaining four tiers. Safety can include any potential assets created as a result of the game. Even though they may not be tangible, have the potential to consume non-trivial amounts of actual time and effort to accumulate, thus providing a real sense of loss aversion if they are put at risk. 


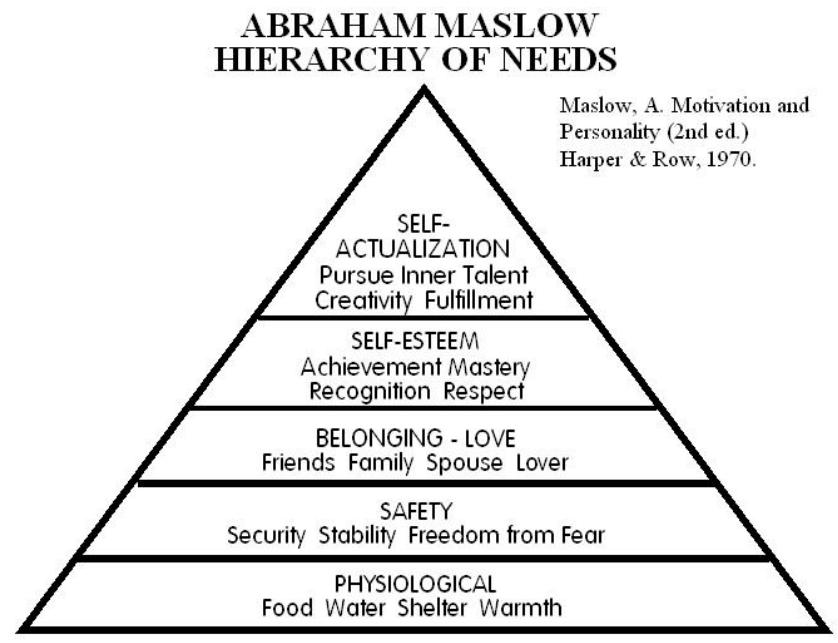

Fig. 3. Maslow's Hierarchy of Needs (Maslow 1970)

The elements of Belonging, friends, family, and intimacy, can be brought about though any game where cooperation is possible. Games are particularly good at Self-Esteem, naturally encouraging achievement and mastery while also providing a medium through which status can be displayed and recognized. The final tier, Self-Actualization, can be accessed via mechanics that offer self-expression or the opportunity for emergent gameplay.

\section{Future Work}

Judging by any substantial collection of players, it is clear that there are many per-sonality types represented, and by nearly any model of personality model, we can see that different types of people respond more or less strongly to various types of needs and motivations. A very interesting area for future development would be identifying which needs and motivations were most strongly represented by each personality type. Furthermore, identifying these types dynamically ingame and altering the gameplay experience accordingly could provide a leap in advancement for many games.

\section{References}

1. Bartle, R.A.: Designing Virtual Worlds. New Rider Publishing, Indianapolis (2003)

2. Maslow, A.: Motivation and Personality. Harper \& Row (1970) 